\title{
Le polymorphisme des onchocercoses humaines ouest-africaines
}

\author{
par A. PROST \\ Organisation Mondiale de la Santé, B.P. 549, Ouagadougou, Haute-Volta.
}

RESUME. 4882 individus, habitant 23 villages dans 8 régions différentes d'Afrique de l'Ouest ont été examinés sur le plan clinique, ophtalmologique et parasitologique en matière d'onchocercose. La maladie sévit sur le mode hyperendémique dans l'ensem. ble de l'échantillon et les différences sont peu sensibles si l'on compare les indices quantitatifs de l'infection par Onchocerca volvulus. Par contre, les complications de la maladie, ophtalmologiques, cutanées et lymphatiques, sont infiniment moins fréquentes dans les quatre grappes situées en forêt que dans celles de savane. Les régions où la maladie est relativement bien tolérée paraissent aussi être celles où les espèces Simulium damnosum s.s. et Simulium sirbanum n'interviennent pas comme vecteurs. L'existence probable de plusieurs complexes vecteur-parasite associés à des manifestations pathologiques très différentes pourraît remettre en cause l'unité taxonomique d'Onchocerca volvulus, ou du moins permettre de caractériser des souches parasitaires.

\section{The different types of human onchocerciasis in west Africa.}

SUMMARY. This study reports the clinical, ophtalmological and parasitological examination for onchocerciasis in 4882 people, living in 23 villages located in 8 different areas of west Africa. The disease is hyperendemic in the whole sample and no major differences occur when comparing the indices of Onchocerca volvulus infection. However, all the complications (eye, skin and lymph node lesions) are less common in the four forest clusters than in to the four savanna clusters. The disease seems to be relatively well tolerated in areas where transmission of Onchocerca volvulus is carried out by Simulium species other than S. damnosum s.s. and S. sirbanum. The striking epidemiological differences of onchocerciasis is probably associated with several vector-parasite complexes, which may indicate the existence of different strains of $\mathrm{O}$. volvulus.

Accepté le 21 novembre 1979. 
La mise en place depuis 1974 du Programme de lutte contre l'Onchocercose dans le Bassin de la Volta, couvrant en totalité ou en partie sept états d'Afrique de l'Ouest, a permis la réalisation d'enquêtes épidémiologiques sur une échelle beaucoup plus vaste que celles qui avaient été entreprises précédemment dans ce domaine. La totale standardisation des examens pratiqués par un nombre réduit d'observateurs a permis également de disposer de résultats strictement comparables dans toute l'aire du Programme.

C'est ainsi qu'il est possible aujourd'hui de préciser les caractères de l'onchocercose dans différentes régions bio-climatiques; l'opposition entre la gravité des symptômes dans la zone des savanes et leur bénignité en zone forestière confirme de façon particulièrement nette la dualité épidémiologique décrite au Cameroun et au Nigéria (1-2-7). Les divergences constatées excèdent de simples variations géographiques et obligent à abandonner la conception unitaire de la maladie.

\section{Cadre de l'étude et méthodes.}

Nous avons sélectionné pour cette étude 23 villages hypérendémiques où la totalité de la population a été examinée entre 1976 et 1979 sur les plans clinique, parasitologique et ophtalmologique selon un protocole normalisé (4-6).

Ces villages sont répartis dans trois états et huit bassins fluviaux et dans des zones où la transmission est due conjointement ou isolément aux six vecteurs principaux ouest-africains d'onchocercose.

Pour assurer une certaine homogénéité de l'échantillon, le recensement a exclu les non-résidents et les familles immigrées depuis cinq ans ou moins dans le village. Chaque individu examiné a subi une double biopsie iliaque (pince de modèle Holth calibre $3 \mathrm{~mm}$ ) où ont été dénombrées les micro-filaires émergées après 30 minutes d'incubation en eau distillée, ou après 24 heures en sérum physiologique si le premier examen était négatif. La micro-filarurie a été recherchée par filtration de $20 \mathrm{ml}$ d'urine sur membrane Millipore ${ }^{(R)}$. L'examen ophtalmologique a intéressé tous les sujets de cinq ans et plus et comporté pour les deux yeux séparément une mesure de l'acuité visuelle; un examen du segment antérieur au biomicroscope oculaire après abaissement de la tête pendant une minute ; un examen du fond d'œil à l'ophtalmoscope ; un exament du champ visuel dans certains cas de lésions postérieures.

Pour simplifier les comparaisons, les 4882 individus examinés ont été regroupés en huit unités d'analyse, ou grappes statistiques, sur la base de la proximité géographique.

D'un côté, ont été regroupés seize villages appartenant à des bassins fluviaux où la transmission est uniquement assurée par les espèces Simulium damnosum sensu stricto et Simulium sirbanum. Il s'agit des villages voltaïques de Mouvielo, Kimpeo, Hemkoa, Kourougbele et Yabar dans le bassin de la Bougouriba, affluent de la Volta noire ; de Niarba, Yakala, Foungou et Bonga dans le bassin de la Volta blanche; des villages nigériens de Molli, Tapoa, Bangou-Koare et Tamou-Kaïna, situés sur des 
affluents temporaires de rive droite du Niger dans l'arrondissement de Say ; enfin des villages de Fétékro, Longo et Sirakoro situés respectivement dans le haut bassin du Nzi, du Bandama et de la Comoé en Côte-d'Ivoire.

Aux fins de comparaison, nous avons retenu sept villages appartenant à la zone forestière : Sioba, Oua et Nimpleu sont dans le bassin du Haut Cavally à la frontière ivoiro-guinéenne ; la transmission y est habituellement due à Simulium yahense mais on a par périodes relevé la présence de Simulium squamosum. Dans les autres villages ivoiriens, Assemanou et Blekoum sur la basse Comoé, Bagro sur le moyen Sassandra, et Galegoua au confluent du Nzi et du Bandama, la transmission est due aux deux espèces Simulium soubrense et Simulium sanctipauli. Ces villages forestiers sont donc situés hors de l'aire de répartition des vecteurs de savane de l'onchocercose.

\section{Résultats.}

Les différentes grappes sont toutes hyperendémiques et il y a peu de différence entre elles en ce qui concerne les index quantitatifs de l'infection par $O$. volvulus : (tableau I) prévalence du parasitisme, densité microfilarienne, taux des porteurs de kystes ont une valeur moyenne légèrement inférieure en forêt qu'en savane. De même, la prévalence du parasitisme oculaire (tableau $I I$ ) un peu plus faible en forêt, reste en relation étroite avec les autres index parasitaires. Globalement, ainsi que l'indiquent les moyennes pondérées, les deux groupes ne sont pas identiques et l'échantillon forestier est significativement moins infecté que l'échantillon de savane. Toutefois, l'étendue qui sépare dans chaque groupe les valeurs extrêmes est suffisamment vaste pour que certaines grappes, également infectées, soient directement comparables.

Sur le plan ophtalmologique (tableau II), 10 à $30 \%$ de la population souffre en savane de lésions onchocerquiennes graves et irréversibles, tandis qu'en forêt ce taux ne dépasse pas $4 \%$ chez les hommes et $2 \%$ chez les femmes. Cette différence est due non seulement à la kératite sclérosante et à l'iridocyclite synéchiante, infiniment moins fréquentes en forêt comme l'avaient constaté Anderson (1) et Budden (2), mais aussi de façon significative aux lésions du segment postérieur, atrophie optique et choriorétinite, alors qu'elle ne l'était pas de façon nette dans les travaux de ces auteurs. La cécité, conséquence directe de la fréquence et du degré d'évolution des lésions graves, atteint 5 à $10 \%$ de la population en savane, moins de $2 \%$ en forêt.

L'origine onchocerquienne de ces cécités est exceptionnelle en forêt (1 cas sur 24) alors qu'en savane elle constitue le facteur étiologique de plus de deux tiers des cas.

La dépigmentation cutanée (vitiligo prétibial) dans les deux sexes et les lésions de dermatite chez l'homme sont plus fréquentes en savane (tableau I). L'interprétation des symptômes "Aine pendante et lymphadénopathie " est plus délicate et la différence géographique moins tranchée. Ces observations nous écartent des constatations nigérianes et camerounaises (1.7) qui font état d'une fréquence plus grande de ces symptômes en forêt. 
La microfilarurie a été recherchée dans les seuls villages forestiers. Son taux est très faible dans le bassin du Cavally $(0$ à $3 \%)$ comme l'avait déjà observé Picq (5). Il augmente sur la basse Comoé : $5 \%$ à Assémanou, $12 \%$ à Blékoum. Ce symptôme n'a pas été recherché au cours de toutes les enquêtes, mais les valeurs moyennes obtenues en Haute-Volta ou au nord Ghana atteignent les taux de 20 à $30 \%$ d'excréteurs de microfilaires urinaires. Il semble que la microfilarurie soit un signe de gravité dont la fréquence est associée à celle des complications oculaires ( $\mathrm{Ba}$ et Rolland, étude en cours). A ce titre, elle constitue en forêt un signe de la meilleure tolérance du parasite.

\section{Commentaires.}

Selon le schéma classique, l'onchocercose de forêt s'opposerait à celle de savane par la fréquence plus grande des kystes, des densités micro-filariennes plus élevées, beaucoup plus de vitiligo et d'aines pendantes, moins de lésions de dermatite, moins de lésions oculaires graves, beaucoup moins d'aveugles. Nous devons ici réformer ce schéma. En effet, les différences bio-géographiques (Forêt-Savane) n'entraînent pas de différences sensibles dans l'infection parasitaire quantitative par $O$. volvulus au sein de populations soumises à une forte transmission.

Mais tous les symptômes de la maladie, et à fortiori les complications, sont beaucoup plus rares en forêt. Dans le cas du foyer du Cavally, malgré une infection très intense, les diverses complications frappent moins de $4 \%$ de la population. Le niveau d'infection un peu plus faible constaté dans l'échantillon de forêt ne saurait être une explication suffisante à l'ampleur des variations observées dans la prévalence des complications.

L'opposition des deux faciès épidémiologiques se fait de façon nette entre le nord, ou la maladie est sévère, et le sud où elle est bien tolérée, de part et d'autre de la frontière géographique assez floue séparant la savane de la forêt. Ces deux biotopes fixent à juste titre la terminologie en usage.

La présente étude, montre que le faciès forestier se rencontre partout où la transmission de la maladie n'est due ni à Simulium damnosum sensu stricto, ni à Simulium sirbanum. Il n'y a par contre aucune différence épidémiologique entre les localités où interviennent uniquement les vecteurs $S$. yahense, $S$. soubrense et $S$. sanctipauli. La forme grave de l'onchocercose paraît donc associée étroitement à la transmission du parasite par les espèces $S$. damnosum et $S$. sirbanum, vecteurs dominant en savane et pénétrant en saison sèche assez profondément vers le sud. La distribution de l'onchocercose forestière pourrait dépendre de la répartition des écosystèmes hostiles à l'implantation des deux espèces de simulies de savane.

Il existe donc ici au moins deux complexes vecteurs(s)-parasite(s) au sens de l'hypothèse de Duke (3). Il est possible qu'à l'instar de Simulium damnosum s.l., qui s'est avérée en Afrique de l'ouest un complexe de huit espèces aujourd'hui isolées, on réussisse à caractériser deux ou plusieurs souches parasitaires d'Onchocerca volvulus, de pathogénicité différente pour l'homme, et transmises préférentiellement par un ou plusieurs des vecteurs reconnus. 


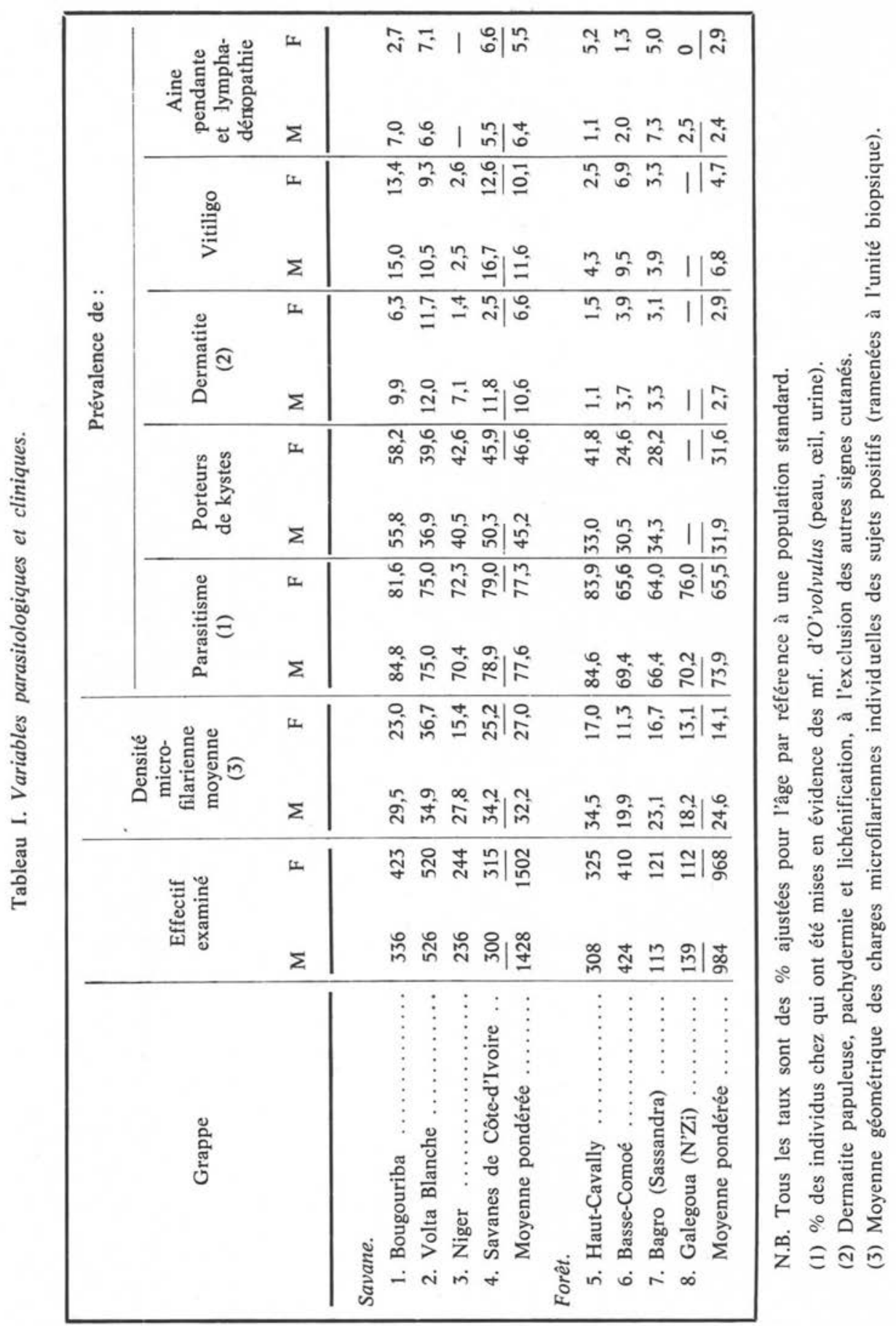




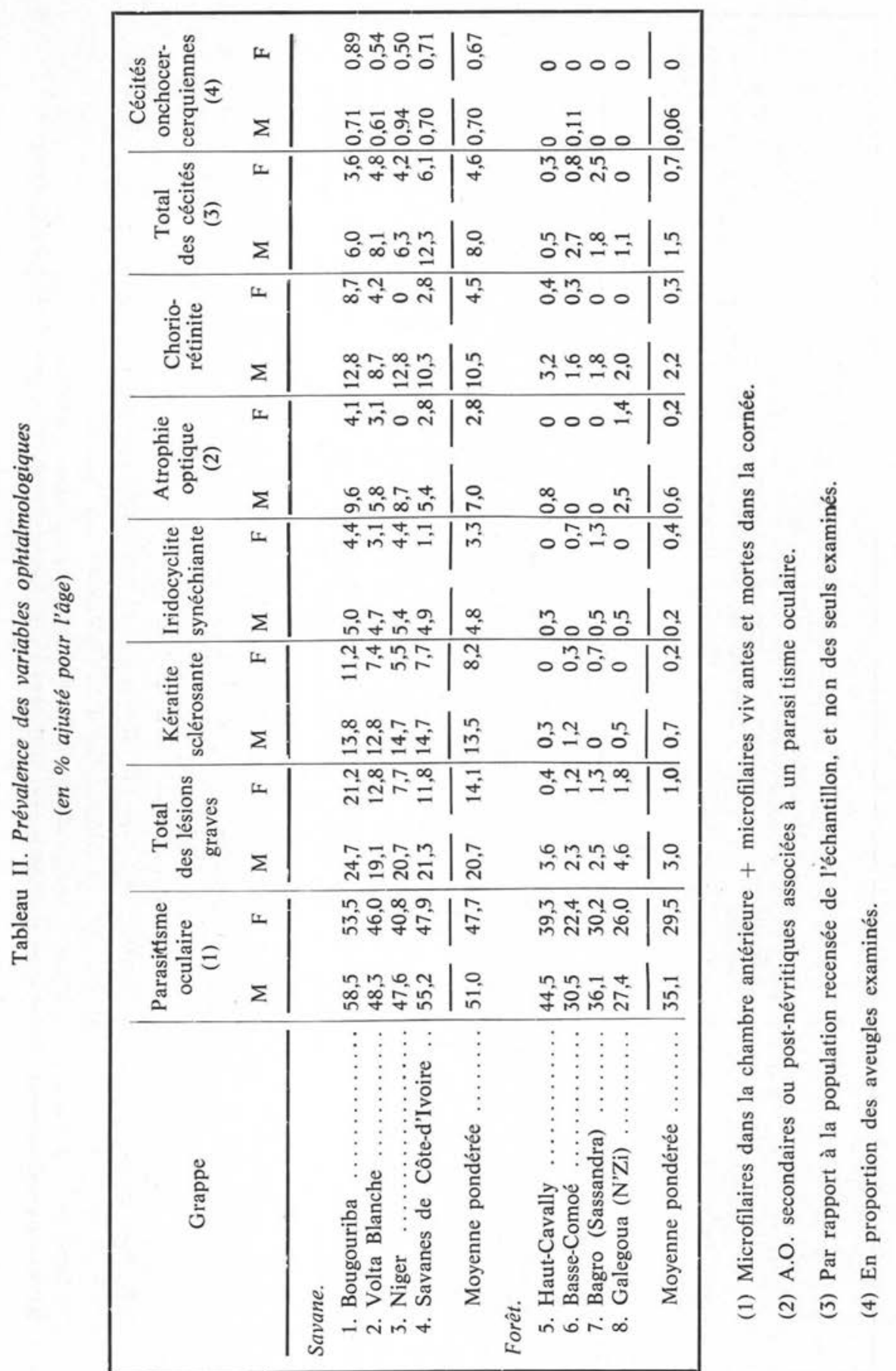


Cette hypothèse n'est qu'un des éléments pouvant expliquer les différences observées. Des facteurs d'environnement et des facteurs d'hôte peuvent aussi intervenir ; l'analyse de l'importance relative de chacun sera discutée dans un autre travail. Mais il est clair dès à présent qu'il faut abandonner une certaine conception unitaire de la maladie.

REMERCIEMENTS. - Nous remercions tous ceux qui ont été impliqués à des titres divers dans les enquêtes épidémiologiques rapportées ici, notamment les docteurs Buttner et Prod'hon, et tout spécialement nos confrères ophtalmologistes les docteurs Thylefors, Tonjum, Rolland et Negrel.

\section{Bibliographie}

1. Anderson J., Fuglsang H., Hamilton P., Marschall T.F. de C.: Studies on onchocerciasis in the United Cameroon Republic. II. Comparison of onchocerciasis in rain-forest and Sudansavanna. Trans. Roy. Soc. Trop. Méd. Hyg., 1974, 68, 209-222.

2. Budden F. H. : Comparative study of ocular onchocerciasis in Savanna and rain-forest. Trans. Roy. Soc. Trop. Méd. Hyg., 1963, 57, 64-70.

3. Duke B. O. L., Lewis J. D., Moore P. J.: Onchocerca-Simulium complexes. 1. Transmission of forest and Sudan-Savanna Strains of Onchocerca volvulus from Cameroon by Simulium damnosum from various west-african bio-climatic zones. Ann. Trop. Méd. Parasitol., 1966, 60, 318-336.

4. Moreau J.-P., Prost A., Prod'hon J.: Essai de normalisation de la méthodologie des enquêtes clinico-parasitologiques sur l'onchocercose en Afrique de l'Ouest. Méd. Trop., 1978, 38, 43-51.

5. Picq J.-J., Roux J.: Sondages préliminaires sur la présence de microfilaires d’Onchocerca volvulus dans les urines de sujets onchocerquiens. Doc. OMS non publié WHO/ONCHO/72.96, 1972, Genève.

6. Prost A., Prod'hon J.: Le diagnostic parasitologique de l'onchocercose. Revue critique des méthodes en usage. Méd. Trop., 1978, 38, 519-532.

7. Woodruff A.W., Anderson J., Pettitt L. E., Tukur M., Woodruff A. H.W.: Some aspects of onchocerciasis in Sudan-Savanna and rain-forest. I. Trop. Med. Hyg., 1977, 80, 68-73. 